\title{
CAPACIDADES DINÂMICAS EM STARTUPS? UMA REVISÃO SISTEMÁTICA
}

\author{
Luisa Tondo Vendruscolo ${ }^{1}$ \\ Marlon Fernandes Rodrigues Alves ${ }^{2}$ \\ Simone Vasconcelos Ribeiro Galina ${ }^{3}$
}

Resumo: Embora os estudos sobre o tema Capacidades Dinâmicas (CD) tenham aumentado nos últimos anos, o foco destes, majoritariamente, está em empresas consolidadas no mercado. Pouco se conhece sobre o desenvolvimento de CD em empresas nascentes, as chamadas startups. Assim o objetivo deste artigo foi realizar uma revisão sistemática sobre $\mathrm{CD}$ em startups. Com os resultados deste trabalho é possível afirmar que há CD sendo desenvolvidas em startups. A contribuição deste trabalho foi o mapeamento da pesquisa sobre CD em startups até o momento e a proposta de uma agenda de pesquisa com base nas lacunas identificadas.

Palavras-chave: Capacidade, Capacidades dinâmicas, Startup, Empreendedorismo

Abstract: Although studies on the Dynamic Capacities (DC) topic have increased in recent years, the focus of these studies is mainly on market consolidated companies. Little is known about DC development in nascent companies, called start-ups. The purpose of this article was to perform a systematic review on DC in start-ups. The result of this work was the indication of the paths about the researches in DC with start-up until now. This way, we could indentify gaps and sugest new directions for this subject.

Keywords: Capacity, Dynamic Capabilities, Start-up, Entrepreneurship

Área: 5.7 Competências e capacitações das empresas

JEL: L25

\footnotetext{
${ }^{1}$ Universidade de São Paulo (USP)

${ }^{2}$ Universidade de São Paulo (USP) e Instituto Federal de Educação, Ciência e Tecnologia de São Paulo (IFSP)

${ }^{3}$ Universidade de São Paulo (USP)
} 


\section{Introdução}

Diversos autores definem as capacidades dinâmicas (CD) em uma série de conceitos, inclusive diferenciando-as em linhas de habilidades e comportamentos ou processos e rotinas (MEIRELLES; CAMARGO, 2014). De acordo com Teece (2012), CD são um elevado nível de competências que determinam a habilidade da firma de se integrar, construir, e reconfigurar recursos/competências internos e externos para buscar e se adequar ao ambiente de negócios que apresentam rápidas mudanças. Esse ambiente em mudança exigiria respostas inovadoras porque, cada vez mais, uma resposta rápida é crítica para a sobrevivência das organizações (TEECE; PISANO; SHUEN,1997).

Os conceitos de ambiente de mudança constante e respostas inovadoras se encontram também na definição de startups mais aceita atualmente. Segundo Blank (2013) startups são empresas inovadoras, em estágio inicial, mas que não podem ser consideradas uma versão menor de uma grande empresa. Essas novas organizações não seguem um plano-diretor ou um plano de negócios como o preconizado para a fundação de empresas tradicionais. Podemos, então, relacionar o conceito de $\mathrm{CD}$ com as startups pela relação de incerteza dessas empresas nascentes.

Com um modelo de negócios ainda a ser validado, as startups estão expostas à diversas alterações antes de se consolidarem como empresas tradicionais. Os ambientes de inovação e empreendedorismo fortalecem o conceito de mudanças rápidas para adequação ao mercado e aos stakeholders. Tendo em vista que as CD foram evidenciadas em empresas consolidadas como um fator estratégico (e.g. ZOLLO ET AL., 2016, HERACLEOUS ET AL., 2016), buscou-se entender neste trabalho os estudos sobre $\mathrm{CD}$ em startups por meio de uma revisão sistemática com o foco em:

- Identificar os artigos que abordam simultaneamente os temas de CD e startups;

- Classificar e codificar as características dos resultados obtidos;

- Fazer um resumo dos trabalhos obtidos nessa revisão;

- Indicar novos caminhos para a pesquisa de CD em startups.

Uma revisão sistemática requer uma pergunta clara, a definição de uma estratégia de busca, o estabelecimento de critérios de inclusão e exclusão dos artigos e, acima de tudo, uma análise criteriosa da qualidade da literatura selecionada (SAMPAIO; MANCINI, 2017). Neste artigo buscou-se responder a pergunta: Qual a relação entre CD e startups?

\section{Referencial teórico}

\subsection{Capacidades dinâmicas}

Segundo o estudo de Meirelles e Camargo (2014) há uma miríade de definições de CD, algumas bastante semelhantes e nota-se fortes controvérsias sobre os condicionantes (antecedentes) e elementos componentes das CD. Há ainda a diferença na visão dos autores que definem $\mathrm{CD}$ como um conjunto de habilidades e capacidades e outros que enfatizam os processos e as rotinas. 
Teece et al. (1997) definem CD como a capacidade para integrar, aprender, reconfigurar e transformar. Especificamente, CD indicam a capacidade de empreendimentos para combinar e coordenar recursos internos e externos, para ganhar e internalizar novos conhecimentos de outras organizações, transformar e reconfigurar a base de recursos em novos processos ou rotinas.

Em outro momento, Teece (2009) define CD como a habilidade da firma em integrar, construir e reconfigurar competências interna e externamente para abordar ambientes em rápida mudança. Por essa definição, competências organizacionais devem ser entendidas como processos organizacionais e gerenciais ou padrões de prática corrente e de aprendizado. A partir desta definição, o autor propõe que existem três capacidades de sustentação das CD: i) capacidade de sentir o contexto do ambiente; ii) capacidade de aproveitar oportunidades; iii) capacidade de gerenciar ameaças e transformações.

Wu (2007) afirma que sem as CD, as empresas que inicialmente são ricas em recursos, podem rapidamente esgotar os investimentos e serem eliminadas. As CD seriam uma forma intermediária para as startups, conhecidas pela limitação de recursos, lidarem em um ambiente dinâmico. Assim, para empreendimentos de base tecnológica, as $\mathrm{CD}$, ou a capacidade de se ajustar a uma rápida mudança do ambiente, é particularmente importante para a sobrevivência.

Para fins de codificação dos resultados deste trabalho adotaremos a definição de Wang e Ahmed (2007). Considerando a visão focada em um conjunto de habilidades e capacidades, definem os elementos componentes das CD como:

i) capacidade adaptativa: é a habilidade da empresa em identificar e capitalizar as oportunidades emergentes de mercado. A capacidade adaptativa reforça a habilidade da organização em se adaptar no tempo certo por meio de flexibilidade dos recursos e alinhamento de seus recursos e suas capacidades com mudanças ambientais;

ii) capacidade absortiva: é a habilidade da empresa em reconhecer o valor de novas informações externas, assimilá-las e aplicar isso para fins comerciais, sendo que a habilidade de avaliar e utilizar conhecimento externo ocorre largamente em função do nível anterior de conhecimento. Isso significa que a capacidade absortiva de uma organização é função de sua trajetória ao longo do tempo. A capacidade absortiva realça a importância de obter conhecimento externo, combiná-lo com conhecimento interno e absorvê-lo para uso interno da organização;

iii) capacidade de inovação: é a habilidade da empresa em desenvolver novos produtos e mercados por meio da orientação do alinhamento estratégico para comportamentos e processos de inovação.

\subsection{Startups}

Segundo Kidder (2012) o termo startup ficou popular no período do "boom" da internet, sendo usado na época como uma maneira de descrever o fenômeno das novas empresas baseadas em tecnologia que surgiam a partir do investimento de capital de risco. Com a difusão das empresas "ponto com" o termo foi ganhando novos contornos e se estabelecendo como uma nova abordagem empresarial especialmente ligada ao empreendedorismo.

No entanto, startups não são somente empresas criadas para a internet. De acordo com Bicudo (2016) startups são mais frequentes na internet porque é menos custoso criar uma empresa online do que uma de agronegócio, por exemplo. O espaço também torna a expansão do negócio bem mais fácil, rápida e barata - além da venda ser repetível e escalável. Hoje o termo extrapola este setor da internet e é aplicável a qualquer iniciativa de novo negócio 
baseado em uma ideia original que tenha características como: rápido crescimento, perfil de risco/recompensa, escalabilidade e busquem liderança de mercado.

Startup significa o ato de começar algo, normalmente relacionado com companhias e empresas que estão no início de suas atividades e que buscam explorar atividades inovadoras no mercado. Gartner (1985) referiu-se a esse processo de criação de uma startup como eventos que acontecem antes de uma organização se tornar uma organização, ou seja, a criação envolve fatores que influenciam o processo de iniciar um negócio.

Startup são empresas jovens e buscam a inovação em qualquer área ou ramo de atividade, procurando desenvolver um modelo de negócio escalável e que seja repetível. São empresas de pequeno porte, recém-criadas ou ainda em fase de constituição, com atividades ligadas à pesquisa e desenvolvimento de ideias inovadoras, cujos custos de manutenção são baixos, oferecendo a possibilidade de rápida e consistente geração de lucros. Essas empresas, normalmente de base tecnológica, possuem espírito empreendedor e uma constante procura por um modelo de negócio inovador.

Uma das definições mais recentes e aceitas é a de Ries (2012) que define uma startup é uma instituição de pessoas tentando criar algo novo sob condições de extrema incerteza. São essas condições de um ambiente de incertezas que aproximam o assunto de startups às CD.

\section{Método}

A metodologia aplicada nesta pesquisa para a coleta de dados foi revisão sistemática. $O$ processo de desenvolvimento desse tipo de estudo de revisão incluiu a caracterização de cada artigo selecionado, identificando-se os conceitos importantes e concluindo com as informações da literatura em relação à determinada intervenção, também se apontou para problemas/questões que sugerem de novos estudos.

\subsection{Coleta de dados}

Definiu-se a estratégia de busca em duas importantes bases de dados - Web of Science e Scopus - com as palavras startup OR start-up OR startups OR start-ups AND "dynamic capability" OR "dynamic capabilities" para que se apresentassem juntas nos documentos. Não foi limitado o período de tempo.

Limitou-se a pesquisa por tipo de documento, selecionando somente artigos e pelos filtros de "Management" e "Business, Management and Accounting", pois as CD derivam de um campo de estudos da Estratégia, que pertence à área de Adminstração. Após a aplicação destes filtros foram encontrados 47 resultados na base de dados Web of Science e 17 artigos na base Scopus. Com o recurso de biblioteca do programa EndNote foram eliminados 10 resultados duplicados.

Dos 54 artigos que apresentaram as duas palavras concomitantemente em qualquer parte do título, resumo ou palavras-chave fez-se um levantamento dos dados como: autores, título do artigo, ano de publicação, revista de publicação, universidade dos autores, país do estudo, resultado encontrado, palavras-chaves.

Foi estabelecido o critério de exclusão de todos os resultados não pertencentes ao estudo da Administração e com a leitura de todos os resumos desses artigos foi possível excluir 8 dos trabalhos. Ao final restaram 46 artigos que satisfizeram as definições e, assim, tiveram seus dados analisados pelo método da análise de conteúdo.

\subsection{Classificação e codificação}


Para a análise de conteúdo foram estabelecidas informações-chave para a classificação e codificação dos resultados. Baseado nos trabalhos de Mariano et al. (2015), Amui et al (2016) foi utilizada a seguinte codificação:

Tabela 1 - Codificação para avaliação da bibliografia selecionada

\begin{tabular}{|c|c|c|}
\hline \multirow{5}{*}{ Origem } & América & $1 \mathrm{~A}$ \\
\hline & Europa & $1 \mathrm{~B}$ \\
\hline & Ásia & $1 \mathrm{C}$ \\
\hline & África & $1 \mathrm{D}$ \\
\hline & Oceania & $1 \mathrm{E}$ \\
\hline \multirow{2}{*}{ Contexto nacional } & Países desenvolvidos & $2 \mathrm{~A}$ \\
\hline & Países em desenvolvimento & 2B \\
\hline \multirow{3}{*}{ Metodologia } & Estudo qualitativo & $3 \mathrm{~A}$ \\
\hline & Estudo quantitativo & $3 \mathrm{~B}$ \\
\hline & Estudo teórico & $3 \mathrm{C}$ \\
\hline \multirow{2}{*}{ Setor estudado } & Alta/média tecnologia & $4 \mathrm{~A}$ \\
\hline & Baixa/nenhuma tecnologia & 4B \\
\hline \multirow{2}{*}{ Foco no tema } & Capacidades dinâmicas como tema principal & $5 \mathrm{~A}$ \\
\hline & Capacidades dinâmicas como tema de suporte & $5 \mathrm{~B}$ \\
\hline \multirow{3}{*}{$\begin{array}{c}\text { Elemento de CD } \\
\text { (WANG; AHMED, } \\
\text { 2007) }\end{array}$} & Capacidade adaptativa & $6 \mathrm{~A}$ \\
\hline & Capacidade de absorção & $6 \mathrm{~B}$ \\
\hline & Capacidade de inovação & $6 \mathrm{C}$ \\
\hline
\end{tabular}

\section{Resultados}

Após a análise do conteúdo completa dos artigos, identificou-se que dos 46 artigos selecionados nas pesquisas às bases de dados Scopus e Web of Science, 31 não abordaram os temas capacidade dinâmica e startup em conjunto. Diversos artigos (TEUBAL, M. ;AVNIMELECH, G.,2003; ANDRÉN, L., MAGNUSSON, M., SJÖLANDER, S., 2003) trouxeram o conceito de CD apenas para sugerir o ambiente de mudança em que as empresas estão inseridas. Outros ainda (BLESA, A.; RIPOLLES, 2008; FOSS, N. J.; LYNGSIE, J., 2014) não focaram o estudo do trabalho em startups e sim em empresas consolidadas.

Portanto, a partir dessa análise, foi possível identificar 15 artigos que de fato conectam os tema de CD e startups (Tabela 2). A primeira publicação unindo os assuntos CD e startup é de 2007 (Tabela 2) e nos últimos três anos do período considerado neste artigo (2014 e 2016) foi produzida a mais de metade dos resultados, ou seja, 8 dos 15 artigos relacionados.

Tabela 2 - Artigos utilizados na revisão sistemática

\begin{tabular}{|c|c|c|c|c|}
\hline Título do Artigo & Autores & Periódico & Ano & Origem \\
\hline $\begin{array}{l}\text { Entrepreneurial resources, dynamic } \\
\text { capabilities and start-up performance of } \\
\text { Taiwan's high-tech firms }\end{array}$ & Wu, L. Y. & $\begin{array}{l}\text { Journal of Business } \\
\text { Research }\end{array}$ & 2007 & Taiwan \\
\hline $\begin{array}{l}\text { Corporate Venture Capital as a Window } \\
\text { on New Technologies: Implications for } \\
\text { the Performance of Corporate Investors } \\
\text { When Acquiring Startups }\end{array}$ & $\begin{array}{l}\text { Benson, } \quad \text { D. } \\
\text {;Ziedonis, R. H. }\end{array}$ & Organization Science & 2009 & EUA \\
\hline The roles of R\&D in new firm growth & $\begin{array}{l}\text { Stam, } \\
\text { E. ;Wennberg, K. }\end{array}$ & $\begin{array}{ll}\text { Small } & \text { Business } \\
\text { Economics } & \end{array}$ & 2009 & $\begin{array}{l}\text { Holanda/ } \\
\text { Reino }\end{array}$ \\
\hline
\end{tabular}




\begin{tabular}{|c|c|c|c|c|}
\hline & & & & Unido \\
\hline $\begin{array}{llr}\text { New Plant } & \text { Venture } & \text { Performance } \\
\text { Differences } & \text { Among } & \text { Incumbent, } \\
\text { Diversifying, and Entrepreneurial } \\
\text { Firms: The Impact of Industry Learning } \\
\text { Intensity }\end{array}$ & $\begin{array}{l}\text { Balasubramanian, } \\
\text { N. }\end{array}$ & Management Science & 2011 & EUA \\
\hline 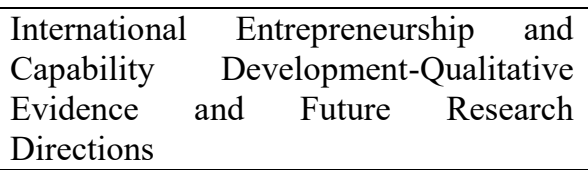 & $\begin{array}{l}\text { Autio, E. ;George, } \\
\text { G. ;Alexy, O. }\end{array}$ & $\begin{array}{l}\text { Entrepreneurship } \\
\text { Theory and Practice }\end{array}$ & 2011 & $\begin{array}{l}\text { Reino } \\
\text { Unido }\end{array}$ \\
\hline $\begin{array}{l}\text { Exploring IT entrepreneurs' dynamic } \\
\text { capabilities using Q-technique }\end{array}$ & Chang, C. C. & $\begin{array}{l}\text { Industrial } \\
\text { Management \& Data } \\
\text { Systems }\end{array}$ & 2012 & Taiwan \\
\hline $\begin{array}{l}\text { Innovation, entrepreneurial activity and } \\
\text { competitiveness at a sub-national level }\end{array}$ & \begin{tabular}{l}
\multicolumn{3}{l}{ Gonzalez-Pernia, } \\
J. L. $\quad$;Pena- \\
Legazkue, I. \\
;Vendrell-Herrero, \\
F.
\end{tabular} & $\begin{array}{ll}\text { Small } & \text { Business } \\
\text { Economics } & \end{array}$ & 2012 & Espanha \\
\hline $\begin{array}{l}\text { How do entrepreneurs know what to } \\
\text { do? learning and organizing in new } \\
\text { ventures }\end{array}$ & $\begin{array}{l}\text { Aldrich, } \\
\text { E. ;Yang, T. T. }\end{array}$ & $\begin{array}{l}\text { Journal of } \\
\text { Evolutionary } \\
\text { Economics }\end{array}$ & 2014 & EUA \\
\hline $\begin{array}{llr}\text { What happens to international } & \text { new } \\
\text { ventures beyond } & \text { start-up: } & \text { An } \\
\text { exploratory study } & & \\
\end{array}$ & $\begin{array}{lrl}\text { Turcan, } & \text { R. } & \text { V. } \\
\text {;Juho, A. } & & \end{array}$ & $\begin{array}{l}\text { Journal } \\
\text { International } \\
\text { Entrepreneurship }\end{array}$ & 2014 & $\begin{array}{l}\text { Dinamarca/ } \\
\text { Finlândia }\end{array}$ \\
\hline $\begin{array}{l}\text { Innovation in start-ups: Ideas filling the } \\
\text { void or ideas devoid of resources and } \\
\text { capabilities? }\end{array}$ & $\begin{array}{l}\text { Paradkar, } \\
\text { A. ;Knight, } \\
\text { J. ;Hansen, P. }\end{array}$ & Technovation & 2015 & $\begin{array}{l}\text { Nova } \\
\text { Zelândia }\end{array}$ \\
\hline $\begin{array}{l}\text { The process of dynamic capability emer } \\
\text { gence in technology startups - } \\
\text { an exploratory longitudinal study in } \\
\text { China }\end{array}$ & $\begin{array}{l}\text { Ma, X.a, Zhou, Z. } \\
\text { b, Fan, X.c }\end{array}$ & $\begin{array}{l}\text { Technology Analysis } \\
\text { and Strategic Manage } \\
\text { ment }\end{array}$ & 2015 & China \\
\hline $\begin{array}{l}\text { The impact of pre-startup planning on } \\
\text { the strength of planning assumptions } \\
\text { and the mode of processing. }\end{array}$ & $\begin{array}{ll}\text { Thiele } & \text { P., } \\
\text { Fellnhofer K. }\end{array}$ & $\begin{array}{l}\text { International Journal } \\
\text { of Entrepreneurial } \\
\text { Venturing }\end{array}$ & 2015 & $\begin{array}{l}\text { Alemanha/ } \\
\text { Liechtenstei } \\
\mathrm{n}\end{array}$ \\
\hline $\begin{array}{l}\text { 'Reverse internationalization' in } \\
\text { Chinese firms: a study of how global } \\
\text { startup OEMs seek to compete } \\
\text { domestically }\end{array}$ & $\begin{array}{l}\text { Chin, T. ;Liu, R. } \\
\text { H. ;Yang, X. }\end{array}$ & $\begin{array}{l}\text { Asia Pacific Business } \\
\text { Review }\end{array}$ & 2016 & China \\
\hline $\begin{array}{l}\text { Strategic Alliances of Entrepreneurial Fi } \\
\text { rms: Value Enhancing Then Value } \\
\text { Destroying }\end{array}$ & $\begin{array}{l}\text { Moghaddam, } \\
\text { K; Bosse, } \\
\text { DA; Provance, M }\end{array}$ & $\begin{array}{l}\text { Strategic } \\
\text { Entrepreneurship } \\
\text { Journal }\end{array}$ & 2016 & EUA \\
\hline $\begin{array}{l}\text { New venture high growth in high-tech } \\
\text { environments }\end{array}$ & $\begin{array}{l}\text { Savarese, M. F; } \\
\text { Orsi, L.; Belussi, } \\
\text { F. }\end{array}$ & $\begin{array}{ll}\text { European } & \text { Planning } \\
\text { Studies } & \end{array}$ & 2016 & Itália \\
\hline
\end{tabular}

A classificação e a categorização destes 16 resultados (Tabela 3) foram realizadas de acordo com os itens da Tabela 1, propostos na metodologia.

Tabela 3 - classificação pela codificação proposta

\begin{tabular}{|c|c|c|c|c|c|c|c|}
\hline $\mathbf{N}^{\mathbf{0}}$ & Título do artigo & Origem & $\begin{array}{c}\text { Contexto } \\
\text { nacional }\end{array}$ & $\begin{array}{c}\text { Metodolo } \\
\text { gia }\end{array}$ & $\begin{array}{c}\text { Setor } \\
\text { estuda } \\
\text { do }\end{array}$ & $\begin{array}{c}\text { Foco } \\
\text { no } \\
\text { tema }\end{array}$ & $\begin{array}{c}\text { Ele } \\
\text { mento } \\
\text { de CD }\end{array}$ \\
\hline 1 & $\begin{array}{c}\text { Entrepreneurial resources, dynamic capabilities } \\
\text { and start-up performance of Taiwan's high-tech } \\
\text { firms }\end{array}$ & $1 \mathrm{C}$ & $2 \mathrm{~B}$ & $3 \mathrm{~B}$ & $4 \mathrm{~A}$ & $5 \mathrm{~A}$ & $6 \mathrm{~B}$ \\
\hline
\end{tabular}




\begin{tabular}{|c|c|c|c|c|c|c|c|}
\hline 2 & $\begin{array}{l}\text { Corporate Venture Capital as a Window on New } \\
\text { Technologies: Implications for the Performance } \\
\text { of Corporate Investors When Acquiring Startups }\end{array}$ & $1 \mathrm{~A}$ & $2 \mathrm{~A}$ & 3B & $4 \mathrm{~A}$ & 5B & $6 \mathrm{~B}$ \\
\hline 3 & The roles of $R \& D$ in new firm growth & 1B & $2 \mathrm{~A}$ & 3B & $4 \mathrm{~A}$ & $5 \mathrm{~B}$ & $6 \mathrm{~B}$ \\
\hline 4 & $\begin{array}{l}\text { New Plant Venture Performance Differences } \\
\text { Among Incumbent, Diversifying, and } \\
\text { Entrepreneurial Firms: The Impact of Industry } \\
\text { Learning Intensity }\end{array}$ & $1 \mathrm{~A}$ & $2 \mathrm{~A}$ & 3B & $4 \mathrm{~A}$ & $5 \mathrm{~B}$ & $6 \mathrm{~A}$ \\
\hline 5 & $\begin{array}{l}\text { International Entrepreneurship and Capability } \\
\text { Development-Qualitative Evidence and Future } \\
\text { Research Directions }\end{array}$ & 1B & $2 \mathrm{~A}$ & $3 \mathrm{~A}$ & $4 \mathrm{~A}$ & $5 \mathrm{~A}$ & $6 \mathrm{~A}, 6 \mathrm{~B}$ \\
\hline 6 & $\begin{array}{l}\text { Exploring IT entrepreneurs' dynamic capabilities } \\
\text { using Q-technique }\end{array}$ & $1 \mathrm{C}$ & $2 \mathrm{~B}$ & $3 \mathrm{~A}$ & $4 \mathrm{~A}$ & $5 \mathrm{~A}$ & $6 \mathrm{~B}$ \\
\hline 7 & $\begin{array}{l}\text { Innovation, entrepreneurial activity and } \\
\text { competitiveness at a sub-national level }\end{array}$ & 1B & $2 \mathrm{~A}$ & 3B & $4 \mathrm{~A}$ & $5 \mathrm{~A}$ & $6 \mathrm{C}$ \\
\hline 8 & $\begin{array}{l}\text { How do entrepreneurs know what to do? learning } \\
\text { and organizing in new ventures }\end{array}$ & $1 \mathrm{~A}$ & $2 \mathrm{~A}$ & $3 \mathrm{C}$ & $4 \mathrm{~A}$ & $5 \mathrm{~B}$ & $6 \mathrm{~B}$ \\
\hline 9 & $\begin{array}{l}\text { What happens to international new ventures } \\
\text { beyond start-up: An exploratory study }\end{array}$ & 1B & $2 \mathrm{~A}$ & $3 \mathrm{~A}$ & $4 \mathrm{~A}$ & $5 \mathrm{~B}$ & $6 \mathrm{~A}$ \\
\hline 10 & $\begin{array}{l}\text { Innovation in start-ups: Ideas filling the void or } \\
\text { ideas devoid of resources and capabilities? }\end{array}$ & $1 \mathrm{E}$ & $2 \mathrm{~A}$ & $3 \mathrm{~A}$ & $4 \mathrm{~A}$ & $5 \mathrm{~B}$ & $6 \mathrm{~B}$ \\
\hline 11 & $\begin{array}{c}\text { The process of dynamic capability emergence in } \\
\text { technology startups - } \\
\text { an exploratory longitudinal study in China }\end{array}$ & $1 \mathrm{C}$ & $2 \mathrm{~B}$ & $3 \mathrm{~A}$ & $4 \mathrm{~A}$ & $5 \mathrm{~A}$ & $6 \mathrm{~A}$ \\
\hline 12 & $\begin{array}{l}\text { The impact of pre-startup planning on the } \\
\text { strength of planning assumptions and the mode } \\
\text { of processing }\end{array}$ & 1B & $2 \mathrm{~A}$ & $3 B$ & $4 \mathrm{~A}$ & $5 \mathrm{~B}$ & $6 \mathrm{~B}$ \\
\hline 13 & $\begin{array}{l}\text { 'Reverse internationalization' in Chinese firms: a } \\
\text { study of how global startup OEMs seek to } \\
\text { compete domestically }\end{array}$ & $1 \mathrm{C}$ & $2 \mathrm{~B}$ & $3 B$ & $4 B$ & $5 \mathrm{~A}$ & $6 \mathrm{~A}, 6 \mathrm{~B}$ \\
\hline 14 & $\begin{array}{l}\text { Strategic Alliances of Entrepreneurial Firms: } \\
\text { Value Enhancing Then Value Destroying }\end{array}$ & $1 \mathrm{~A}$ & $2 \mathrm{~A}$ & $3 B$ & $4 \mathrm{~A}$ & $5 \mathrm{~A}$ & $6 \mathrm{~B}$ \\
\hline 15 & $\begin{array}{l}\text { New venture high growth in high-tech } \\
\text { environments }\end{array}$ & 1B & $2 \mathrm{~A}$ & $3 B$ & $4 \mathrm{~A}$ & $5 \mathrm{~B}$ & $6 \mathrm{~B}$ \\
\hline
\end{tabular}

\subsection{Origem}

De acordo com os critérios estabelecidos na metodologia, pode-se observar que as origens dos autores, conforme informado às bases de dados, são diversas (tabela 2). Os autores dos países Holanda, Alemanha, Reino Unido, Dinamarca, Itália, Espanha e Nova Zelândia apresentaram apenas um artigo cada. China, Taiwan respondem por 2 artigos cada e os Estados Unidos por 4. Portanto, pode-se notar que até o momento a produção bibliográfica, abordando os temas CD e startups, está em países desenvolvidos, notadamente nos Estados Unidos. Em termos de parcerias, apenas três artigos apresentaram de autores de diferentes países.

\subsection{Contexto nacional}


Quanto ao contexto nacional é possível afirmar que apenas quatro estudos foram realizados em países em desenvolvimento, China e Taiwan. Os 11 demais artigos foram realizados em países desenvolvidos. Além dos quatro artigos em países em desenvolvimento onde os autores pesquisaram em um país diferente da sua origem, apenas os autores do Reino Unido com o trabalho de Autio, George e Alexy (2011), optaram por estudar empresas na Finlândia que internacionalizaram suas operações no início de suas atividades.

\subsection{Metodologia}

Considerando a necessidade de aprofundar os estudos sobre CD em startups, buscou-se definir as metodologias aplicadas nos artigos selecionados. De acordo com a codificação proposta neste artigo é possível afirmar que o método mais utilizado foi o de estudos quantitativos (9 artigos), com aplicação de modelos estatísticos e com o método dos Mínimos Quadrados Ordinários sendo apresentado em seis artigos. A outra metodologia mais utilizada foi qualitativa com 5 artigos e apenas um trabalho teórico na área (ALDRICH, YANG, 2014).

\subsection{Setor estudado}

Apenas um estudo foi realizado em um setor de baixa tecnologia. Chin, Liu e Yang (2016) propuseram um estudo empresas de manufatura, fábricas de trabalho intensivo. Os demais resultados foram em empresas high-tech e sua maioria em empresas de Tecnologia da Informação.

\subsection{Foco no Tema}

Os resultados dessa análise sistemática apontam que sete dos artigos analisados abordam CD como tema principal do estudo. Os outro oito artigos utilizam CD como uma variável de menor importância no modelo proposto ou para indicar novas $\mathrm{CD}$, diferentes das consideradas na análise deste trabalho.

\subsection{Elemento de CD}

De acordo com o conceito de Wang e Ahmed (2007), os elementos das CD são as capacidades de adaptação, capacidade de absorção e capacidade de inovação. A capacidade de absorção (5B) foi a que mais se destacou nos resultados sendo apresentada em 12 artigos. Isso se dá porque diversos artigos trazem o elemento de alianças estratégicas para o conceito de CD. Entende-se que as alianças e a também a aplicação dos recursos dos empreendedores (experiência anterior, networking) estão vinculadas à capacidade de absorção, pois são a aplicação de elementos e conhecimentos externos à startup.

Dos demais artigos, 4 evidenciam a capacidade de adaptação, dos quais 2 indicam a sua ocorrência em concomitância com a capacidade de absorção. Apenas um artigo relata a capacidade de inovação em startups. Para um melhor entendimento sobre os resultados, a Tabela 4 apresenta o resumo dos artigos e as abordagens da CD. 
Tabela 4 - Descrição dos trabalhos

\begin{tabular}{|c|c|c|c|}
\hline & Título do Artigo & Resumo & Abordagem da CD \\
\hline 1 & $\begin{array}{l}\text { Entrepreneurial resources, } \\
\text { dynamic capabilities and start-up } \\
\text { performance of Taiwan's high- } \\
\text { tech firms }\end{array}$ & $\begin{array}{l}\text { Estudo sobre empresas do setor high-tech em Taiwan afirma que } \\
\text { startups não podem ser avaliadas somente por Resouce-based } \\
\text { Review (RBV). O autor sugere que em ambientes dinâmicos os } \\
\text { recursos, somente, não se traduzem em performance e que as CD } \\
\text { fariam essa interligação. Em conclusão, o autor afirma que os } \\
\text { recursos da startup, tanto internos como externos, não } \\
\text { influenciam diretamente o desempenho da startup. A influência } \\
\text { no desempenho se dá por meio de recursos intangíveis } \\
\text { dinâmicos. Outros resultados que também devem ser destacados } \\
\text { são: (1) recursos empresariais abundantes tendem a aumentar a } \\
\text { disponibilidade de entidades externas para entrar em redes de } \\
\text { cooperação com a inicialização da empresa e (2) a eficácia da } \\
\text { rede cooperativa da empresa tende a melhorar as CD da startup. }\end{array}$ & $\begin{array}{l}\text { O trabalho aborda a capacidade de absorção e evidencia que a } \\
\text { capacidade dinâmica de startups é composta por dois componentes } \\
\text { principais: os recursos complementares de parceiros externos e } \\
\text { recursos internos do empreendedor em si. Consequentemente, uma } \\
\text { pré-condição (ou componente) para a startup ser capaz de } \\
\text { combinar, coordenar, transformar e realocar recursos, incluindo } \\
\text { internos e externos, é que esses recursos sejam abundantes. Assim, } \\
\text { conclui-se que a integração, a reconfiguração e a aprendizagem só } \\
\text { se tornam significativas quando os recursos são abundantes . }\end{array}$ \\
\hline 2 & $\begin{array}{l}\text { Corporate Venture Capital as a } \\
\text { Window on New Technologies: } \\
\text { Implications for the Performance } \\
\text { of Corporate Investors When } \\
\text { Acquiring Startups }\end{array}$ & $\begin{array}{l}\text { No artigo sobre aquisição de startups por multinacionais, } \\
\text { abordam a capacidade de absorção, um dos pilares das } \mathrm{CD} \text {, como } \\
\text { fator a ser considerado no momento da aquisição de uma startup. }\end{array}$ & $\begin{array}{l}\text { A capacidade dinâmica é utilizada apenas como uma das variáveis } \\
\text { utilizadas para avaliação da aquisição, porém esse resultado não é } \\
\text { retomado no resultado e conclusão do trabalho. }\end{array}$ \\
\hline 3 & $\begin{array}{l}\text { The roles of R\&D in new firm } \\
\text { growth }\end{array}$ & $\begin{array}{l}\text { Buscando identificar o papel da Pesquisa e Desenvolvimento } \\
\text { (P\&D) no crescimento das empresas, os autores identificam CD } \\
\text { como processos específicos e identificáveis como P\&D, } \\
\text { desenvolvimento de produtos e alianças. As conclusões são que a } \\
\text { P\&D nas empresas high-tech influencia o crescimento através de } \\
\text { alianças entre firmas, possibilitando a exploração de conteúdo } \\
\text { externo. De acordo com esses autores, o investimento em P\&D } \\
\text { estimula o desenvolvimento de produtos das empresas, porém } \\
\text { não o seu crescimento. Por fim, afirmam que o crescimento das } \\
\text { empresas low-tech depende mais da ambição do empreendedor } \\
\text { do que da própria P\&D. }\end{array}$ & $\begin{array}{l}\text { Os resultados apresentam a relação positiva entre P\&D e a geração } \\
\text { de alianças. }\end{array}$ \\
\hline
\end{tabular}


4 Absorptive Capacity and Firm Performance in SMEs: The Mediating Influence of Strategic Alliances

International Entrepreneurship and Capability DevelopmentQualitative Evidence and Future Research Directions

6 Exploring IT entrepreneurs dynamic capabilities using Qtechnique

Innovation, entrepreneurial activity and competitiveness at a sub-national level

8 How do entrepreneurs know what to do? learning and organizing in new ventures
O estudo buscou identificar se a relação entre Capacidade de Absorção e o desempenho da empresa pequenas e médias (PME) é mediada por alianças estratégicas. Para esse autores, as alianças estratégicas têm um grande efeito sobre o desemprenho da firma, enquanto a capacidade de absorção influencia tanto o desempenho da firma como o sucesso das alianças estratégicas.

Em uma transferência de conhecimento com sucesso é essencial a interação entre capacidade de absorção e as alianças estratégicas.

$\mathrm{O}$ artigo explora como novas capacidades emergem e se

solidificam em novos empreendimentos em ambientes de

incerteza. Para isso estudaram sob a lente das capacidades e

cognição empresarial a internacionalização de novos

empreendimentos. Foi desenvolvido um modelo baseado em

cognição para identificar as capacidades encontradas nessa

condição de internacionalização.

Define no artigo quatro CD que os empreendedores de empresas

de Tecnologia da Informação devem apresentar. Ressalta-se que

foi realizada apenas uma análise do ponto sociológico e não

foram estudados como esses fatores afetam o desempenho da

empresa. As quatro CD sugeridas são: sensibilidade orientada

para mercado, habilidade de absorver o conhecimento,

capacidade de networking e a habilidade de comunicar e

negociar.

Usando dados das regiões espanholas, NUTS 2, no período

2000-2004, busca identificar a relação entre as capacidades de

gerar novos conhecimentos e empreender com o nível de

competitividade regional.

\section{A exposição do empreendedor para apreender sugere}

conhecimentos que ele aprenda, desenvolva ou tenha. $\mathrm{O}$ artigo

considera que o empreendedor só aprende de 3 formas: fazendo,

imitando/copiando ou na tentativa e erro. Abordando 3

elementos a serem apreendidos- o hábito, a heurística e a rotina,

o artigo traz que somente a rotina pode ser incorporada pelo

empreendedor de acordo com seu ambiente de trabalho.
Especialmente em no contexto das PME, alianças estratégicas são um meio útil para expandir a base de conhecimento de uma empresa rapidamente e com eficiência nos custos, porque os riscos e os custos são compartilhados com o parceiro de aliança.

A conclusão do artigo traz que a internacionalização de startups fo positiva no desenvolvimento de capacidades como o reconhecimento de seus processos internos.

Essas capacidades fazem referência à capacidade de absorção, aprendizagem organizacional, teoria baseada em recursos e teoria do sistema adaptativo complexo, respectivamente.

O artigo considera a geração de conhecimento e a capacidade de empreender como uma capacidade dinâmica. Utiliza o conceito de capacidades dinâmica de Zahra (2006) para tornar dinâmico o modelo proposto de identificação da relação entre inovação e empreendedorismo com a produtividade regional.

A conclusão é que a rotina de processos na startup pode ser aprendida, caracterizando isso como uma capacidade de absorção. 
9 What happens to international new ventures beyond start-up:

An exploratory study

10 Innovation in start-ups:

filling the void or ideas devoid of resources and capabilities?

11 The process of dynamic capabilit y emergence in technology startups -

an exploratory longitudinal stud $\mathrm{y}$ in China

12 The impact of pre-startup planning on the strength of planning assumptions and the mode of processing.

13 'Reverse internationalization' in Chinese firms: a study of how global startup OEMs seek to compete domestically

14 Strategic Alliances of Entreprene urial Firms: Value Enhancing Then Value Destroying
Artigo defende que startups internacionalizadas não atingem made-it point sem CD. Os constructos são a experimentação estratégica, tensões nas gestão e "mentiras legítimas".

$\mathrm{O}$ trabalho investiga como o acesso a diferentes tipos de recursos afetam o sucesso de uma startup. startups de tecnologia no mercado chinês.

O trabalho propõe a analise se um planejamento pré startup tem um impacto negativo nas capacidades cognitivas do empreendedor fundador.

$\mathrm{O}$ artigo busca verificar se as startups de manufatura poderiam aplicar a capacidade de aquisição do conhecimento, que obtiveram a partir processo de internacionalização, para a competição regional. Esse movimento seria a "Reverse internationalization".

Baseado na RBV e CD o artigo se propõe a avaliar seu efeito das alianças na conquista de mercado de empresas da indústria de software antes do IPO.
O trabalho aponta que as "mentiras legítimas" são uma capacidade dinâmica no contexto de uma startup.

Confirmam a segunda proposição do seu artigo que afirma que os recursos baseados na capacidade, especialmente nas $C D$, têm um impacto maior sobre a vantagem competitiva de startups do que ativos tangíveis ou intangíveis. No artigo as CD não foram caracterizadas e apenas trazidas de forma superficial.

Ainda segundo os autores, as startups desenvolvem seus recursos disponíveis (como marcas e patentes, conhecimentos) para formar alianças com empresas que complementem seus recursos.

Como resultado da pesquisa realizada confirmam que as CD de uma startup são diferentes das de firmas bem estabelecidas.

Especificamente, os cinco casos estudados mostram que a capacidade de uma startup em identificar oportunidades (sensing), em última análise, conta com o conhecimento existente e experiência passada empreendedor, que constantemente atualizado, aplica aos negócios em tempo real e com os negócios em andamento.

Utiliza a definição de CD para aplicar no conceito de "modelo mental" que seria um recurso para tomada de decisão. Os autores buscaram relacionar as consequências de haver um plano pré-statup com as $\mathrm{CD}$, aqui tratadas como "cognitive dynamic capabilities".

Apesar de não definirem as $\mathrm{CD}$, validam a hipótese de que a capacidade dinâmica de aquisição do conhecimento, a partir do processo de internacionalização, é positiva para a competição local da empresa, a qual os autores propõem chamar de "Reverse internationalization".

Define alianças estratégicas como uma capacidade dinâmica e conclui que há uma relação positiva e significante para o mercado, porém um grande número de alianças é prejudicial para a valoração dessa empresa. 
15 New venture high growth in high-tech environments
O artigo, através do estudo de uma amostra de startups italianas e Utiliza capacidade dinâmica em um dos modelos estatísticos e

consideradas high-growth, busca identificar os gatilhos de

crescimento dessas empresas.
Utiliza capacidade dinâmica em um dos modelos estatísticos e
conclui que desenvolvimento de $\mathrm{CD}$, considerando investimento

em recursos humanos e novas rotinas, é significativo para o

crescimento da startup. 


\section{Discussão e considerações finais}

A distribuição geográfica da origem dos autores e dos contextos das pesquisa evidenciam a prevalência dos países desenvolvidos na pesquisa de $C D$ em startups. Em que se pese abundância de capital e o elevado desenvolvimento tecnológico dos países desenvolvidos, os países em desenvolvimento constituem uma oportunidade para desenhos de pesquisa que permitam entender sob quais circunstâncias os presentes resultados permanecem válidos. Para além das implicações em termos de generalizações, países em desenvolvimento avançam cada vez mais nos rankings de patenteamento, produção científica e crescimento econômico.

Em termos metodológicos, a lacuna de estudos conceituais seguida de estudos qualitativos é contrastante com a pesquisa em $\mathrm{CD}$ em geral, onde há um número de grande de pesquisas teóricas e o crescimento de pesquisas quantitativas é mais recente. Além do mais, considerando o estágio inicial da pesquisa de CD em startups, é ainda mais flagrante o predomínio de pesquisas quantitativas. Cabe ressaltar que a aplicação de métodos mistos seria uma oportunidade de pesquisa na área.

Já o foco em setores de alta tecnologia possivelmente é viés do entendimento de startups como sinônimo de empresas de TI. A consecução de objetivos estratégicos por meio de processos e rotinas organizacionais não oferece qualquer restrição teórica para pesquisa em setores não tecnológicos, cabe aos pesquisadores pesquisar outras empresas, como as startups de impacto social.

O elevado número de artigos que usam $\mathrm{CD}$ apenas como tema de suporte é um fato que está relacionado com o redução de artigos no filtro inicial de 46 para 15. Diference do cenário metodológico, essa questão se repete na pesquisa em CD em geral, dado o interesse no tema os pesquisadores mencionam CD sem de fato adentrar no assunto. Isso é prejudicial para o desenvolvimento teórico das $\mathrm{CD}$, levando a reificação, um fenômeno no qual um conceito é repetido com significado cada vez mais distantes do original (GIUDICI, REINMOELLER, 2012).

Por fim os elementos das CD enfatizados na pesquisa em startups são surpreendentes. Primeiro, pela ausência de abordagens integrativas que explorem a multidimensionalidade do constructo de $\mathrm{CD}$ nas startups, apenas dois artigos exploraram mais que uma dimensão. Segundo, pela escasso enfoque na capacidade de inovação, no qual apenas um artigo focou, dado que startups têm sua acepção vinculada a solução de um problema para criação de um modelo de negócio escalável. Terceiro, pelo enfoque excessivo no empreendedor, já que uma startup é antes de tudo uma organização social, empreendedor não é sinônimo da mesma. Em suma, essa pesquisa mapeou o estado da arte da pesquisa de CD em startups. Estudos futuros devem contemplar países em desenvolvimento, abordagens qualitativas ou conceituais, foco em setores de baixa tecnologia e privilegiar outras dimensões da CD. Mais importante, devem manter o rigor nos pressupostos teóricos da $\mathrm{CD}$ e o enfoque nas startups e suas idiossincrasias a fim de contribuir com a literatura. 


\section{REFERÊNCIAS}

AMUI, L. B; JABBOURAC. J.; JABBOURA, A. B; KANNANE, D. Sustainability as a dynamic organizational capability: a systematic review and a future agenda toward a sustainable transition. Journal of Cleaner Production, v. 142, Part 1, p.308-322, 2017

ALDRICH, H. E.; YANG, T. T. How do entrepreneurs know what to do? learning and organizing in new ventures. Journal of Evolutionary Economics, v. 24, n. 1, p. 59-82, 2014.

ANDRÉN, L.; MAGNUSSON, M.; SJÖLANDER, S. Opportunistic adaptation in start-up companies. International Journal of Entrepreneurship and Innovation Management, v. 3, n. 56, p. 546-562, 2003.

AUTIO, E.; GEORGE, G.; ALEXY, O. International Entrepreneurship and Capability Development-Qualitative Evidence and Future Research Directions. Entrepreneurship Theory and Practice, v. 35, n. 1, p. 11-37, Jan 2011.

BALASUBRAMANIAN, N. New Plant Venture Performance Differences Among Incumbent, Diversifying, and Entrepreneurial Firms: The Impact of Industry Learning Intensity. Management Science, v. 57, n. 3, p. 549-565, Mar 2011.

BENSON, D.; ZIEDONIS, R. H. Corporate Venture Capital as a Window on New Technologies: Implications for the Performance of Corporate Investors When Acquiring Startups. Organization Science, v. 20, n. 2, p. 329-351, Mar-Apr 2009.

BICUDO, Lucas. Afinal, o que é uma startup?. Startse Infomoney. São Paulo. Disponível em:< http://startse.infomoney.com.br/portal/2016/05/03/18963/afinal-o-que-e-uma-startup/>. Acesso em 10/06/2016.

BLESA, A.; RIPOLLES, M. The influence of marketing capabilities on economic international performance. International Marketing Review, v. 25, n. 6, p. 651-673, 2008.

CHIN, T.; LIU, R. H.; YANG, X. M. 'Reverse internationalization' in Chinese firms: a study of how global startup OEMs seek to compete domestically. Asia Pacific Business Review, v. 22, n. 2, p. 201-219, Apr 2016.

CHANG, C. C. Exploring IT entrepreneurs' dynamic capabilities using Qtechnique. Industrial Management \& Data Systems, v. 112, n. 8-9, p. 1201-1216, 2012.

DA COSTA, P. R.; PORTO, G. S. Technological governance and cooperability in Brazilian multinationals. Rae-Revista De Administracao De Empresas, v. 54, n. 2, p. 201-221, MarApr 2014.

FLATTEN, T. C.; GREVE, G. I.; BRETTEL, M. Absorptive Capacity and Firm Performance in SMEs: The Mediating Influence of Strategic Alliances. European Management Review, v. 8, n. 3, p. 137-152, Fal 2011.

FOSS, N. J.; LYNGSIE, J. The strategic organization of the entrepreneurial established firm. Strategic Organization, v. 12, n. 3, p. 208-215, Aug 2014. 
GARTNER, W. B. "A Conceptual Framework for Describing the Phenomenon of New Venture Creation." The Academy of Management Review, vol. 10, no. 4, 1985, pp. 696706., www.jstor.org/stable/258039.

GIUDICI, Alessandro; REINMOELLER, Patrick. Dynamic capabilities in the dock: A case of reification?. Strategic Organization, v. 10, n. 4, p. 436-449, 2012.

GONZALEZ-PERNIA， J. L.; PENA-LEGAZKUE， I.; VENDRELL-HERRERO, F. Innovation, entrepreneurial activity and competitiveness at a sub-national level. Small Business Economics, v. 39, n. 3, p. 561-574, Oct 2012.

HERACLEOUS, Loizos et al. Structural ambidexterity and competency traps: Insights from Xerox PARC. Technological Forecasting and Social Change, 2016, no prelo.

KIDDER, D. The Startup Playbook: secrets from the fastest-growing startups from their founding entrepreneurs. Chronicle Books LLC, 2012.

MA, Xiaofeng; ZHOU, Zhao; FAN, Xiouhong. The process of dynamic capability emergence in technology start-ups - an exploratory longitudinal study in China. Technology Analysis and Strategic Management, London, v. 27, n. 6, p. 675-692, 2015.

MARIANO, E.B., SOBREIRO, V.A., DO NASCIMENTO REBELATTO, D.A. Human development and data envelopment analysis-A structured literature review. Omega, v. 54, p. 33 e 49. Disponível em < http://dx.doi.org/10.1016/j.omega.2015.01.002> Acesso em 03 de Mar 2017.

MEIRELlES, Dimária Silva e; CAMARGO, Álvaro Antônio Bueno. Capacidades Dinâmicas: O Que São e Como Identificá-las?. Rev. adm. contemp., Curitiba , v. 18, n. spe, p.41-64, Dec.2014.Disponível em $<$ http://www.scielo.br/scielo.php?script=sci_arttext\&pid=S141565552014000700041\&lng=en $\&$ nrm=iso $>$. Acesso em: 30 Ago 2016.

MOGHADDAM.K; BOSSE,D.A; PROVANCE,M.Strategic Alliances of Entrepreneurial Fir ms: Value Enhancing Then Value Destroying Strategic. Entrepreneurship Journal, 10 p.153-168, 2016.

PARADKAR, A.; KNIGHT, J.; HANSEN, P. Innovation in start-ups: Ideas filling the void or ideas devoid of resources and capabilities? Technovation, v. 41-42, p. 1-10, Jul-Aug 2015.

RIES, Eric. A Startup Enxuta: Como os empreendedores atuais utilizam a inovação contínua para criar empresas extremamente bem-sucedidas. Brasil: Leya, 2012.

SAMPAIO, RF; MANCINI, MC.Estudos de revisão sistemática:um guia para síntese criteriosa da evidência científica. Rev. bras. fisioter. 2007, vol.11, n.1, pp.83-89. http://dx.doi.org/10.1590/S1413-35552007000100013.

SAVARESE, M. F; ORSI, L.; BELUSSI, F. New venture high growth in high-tech environments. European Planning Studies, 2016. Disponível em $<$ http://dx.doi.org/10.1080/09654313.2016.1232700> Acesso em 03 de Mar 2017. 
STAM, E.; WENNBERG, K. The roles of R\&D in new firm growth. Small Business Economics, v. 33, n. 1, p. 77-89, Jun 2009.

THIELE, P.; FELLNHOFER, K. The impact of pre-startup planning on the strength of planning assumptions and the mode of processing. International Journal of Entrepreneurial Venturing, v. 7, n. 2, p. 173-193, 2015. Disponível em: < https://www.scopus.com/inward/record.url?eid=2-s2.0-

84926663312\&partnerID=40\&md5=7f9cd239b7b6f51 ec5ae5133ee847b32 >.

TEECE, D. J.; PISANO, G.; SHUEN, A. Dynamic Capabilities and Strategic Management. Strategic Management Journal, Oxford, p. 509-533, 1997.

TEECE, David J. Dynamic Capabilities: Routines versus Entrepreneurial Action. Journal Of Management Studies, Oxford, v. 49, Ed. 8 p.1395-1401, 2012.

TEUBAL, M.; AVNIMELECH, G. Foreign acquisitions and R\&D leverage in High Tech industries of peripheral economies. Lessons and policy issues from the Israeli experiences. International Journal of Technology Management, v. 26, n. 2-4, p. 362-385, 2003. ISSN 0267-5730.

TURCAN, R. V.; JUHO, A. What happens to international new ventures beyond start-up: An exploratory study. Journal of International Entrepreneurship, v. 12, n. 2, p. 129-145, 2014. ISSN $15707385 \quad$ (ISSN). Disponível em: < https://www.scopus.com/inward/record.url?eid=2-s2.084905124534\&partnerID=40\&md5=bbaede0ebbc26c37107035d0a69f9d95 >.

ZAHRA, S. A.; SAPIENZA, H.J.; DAVIDSSON, P. Entrepreneurship and Dynamic Capabilities: A Review, Model and Research Agenda. Journal of Management Studies 43(4):pp. 917-955, 2006.

ZHANG, M. Information systems, strategic flexibility and firm performance: An empirical investigation. Journal of Engineering and Technology Management. v. 22, n. 3, p. 163184, September 2005.

ZOLLO, M.; WINTER, S. G. Deliberate learning and the evolution of dynamic capabilities. Organization science, v. 13, n. November 2013, p. 339-351, 2002.

ZOLLO, Maurizio et al. Toward a Comprehensive Model of Organizational Evolution: Dynamic Capabilities for Innovation and Adaptation of the Enterprise Model. Global Strategy Journal, v. 6, n. 3, p. 225-244, 2016.

WANG, C. L; AHMED, P.K. Dynamic capabilities: A review and research. International Journal of Managemen Reviews, Oxford, v. 9, p.31 - 51, 2007.

WU, L. Y. Entrepreneurial resources, dynamic capabilities and start-up performance of Taiwan's high-tech firms. Journal of Business Research, v. 60, n. 5, p. 549-555, 2007. 